\title{
Pepila Weise: Taxonomic identity of the genus and revision of the megalopoides species-group, and description of two new species from Australia (Coleoptera: Chrysomelidae: Alticinae)
}

\author{
MaUrizio BIONDI and Paola D'ALESSANDRO
}

Dipartimento di Scienze Ambientali, University of L'Aquila, 67010 Coppito (AQ), Italy; e-mail: biondi@univaq.it

Key words. Coleoptera, Chrysomelidae, Pepila, Australia, taxonomy, new species, new combinations

\begin{abstract}
In this paper some taxonomic observations on the Australian flea beetle genus Pepila Weise, 1923 are reported. The following species are transferred to the genus Pepila and lectotypes for them designated: Chaetocnema carinata Baly, 1877; Plectroscelis crassipennis Blackburn, 1896; Chaetocnema fuscomaculata Baly, 1877; Plectroscelis hypocrita Blackburn, 1896; Chaetocnema laticeps Baly, 1877; Plectroscelis meyricki Blackburn, 1896; Dibolia ochracea Waterhouse, 1838; Plectroscelis pallidior Blackburn, 1896; Dibolia pygmaea Waterhouse, 1838; Plectroscelis quadraticollis Blackburn, 1896; Chaetocnema submetallescens Baly, 1877; Plectroscelis tumbyensis Blackburn, 1896; Chaetocnema waterhousei Baly, 1877. The synonymy of Chaetocnema submetallescens Baly, 1877 with Plectroscelis longior Blackburn, 1896 is proposed. In addition, the megalopoides species-group, including P. megalopoides Weise, 1923, P. uptoni n.sp., and P. longifallica n.sp., is analyzed.
\end{abstract}

\section{INTRODUCTION}

The genus Pepila was described by Weise (1923) to accommodate the species Chaetocnema megalopoides Baly, 1877 from Australia. Weise (1923) asserted that "Diese Gattung Pepila ist aufs Nächste mit Chaetocnema verwandt" but did not differentiate the new genus. The most important distinguishing characters identified by Weise (1923) was the presence of a hind globose apical tarsal segment and a head that clearly protrudes from the prothorax.

Based on new characters, we redefine the taxonomic identity of this flea beetle genus. We also transfer to Pepila about forty species, of which 13 were previously included in Chaetocnema Stephens, 1831. The other species are new to science and will be described soon (Biondi \& D'Alessandro, in prep.). In this contribution, the taxonomic position of the genus Pepila is discussed, and the megalopoides species-group including $P$. megalopoides (Baly), P. uptoni n. sp. and P. longifallica n. sp. analyzed.

\section{MATERIALS AND METHODS}

Material consists of preserved dry insects provided by the following institutions: Australia, Australian Capital Territory, Canberra City, CSIRO, Australian National Insect Collection (ANIC); United Kingdom, London, The Natural History Museum [formerly British Museum (Natural History)] (BMNH); Sweden, Stockholm, Naturhistoriska Riksmuseet (NHRS); Australia, South Australia, Adelaide, South Australia Museum (SAM). The specimens were examined using WILD MZ8 and MZ12.5 binocular microscopes. The scanning electron micrographs were taken using a PHILIPS SEM XL30 CP.

Abbreviations. LAED - length of median lobe of aedeagus; LAN - length of antennae; LB - total length of body; LE length of elytra; LP - length of pronotum; LSP - length of spermatheca; WE - width of elytra; WP - width of pronotum.

\section{PEPILA WEISE, 1923}

Pepila Weise, 1923: 126.

Type species. Chaetocnema megalopoides Baly, 1877 by original designation (Weise, 1923: 126).

Diagnosis. Pepila has the following diagnostic characters, which separate it from the genus Chaetocnema Stephens, 1831 (see also Biondi, 2002): a) proximal half of hind tibiae dorsally channelled (Figs 7-8); b) frontal sulci short, or if elongate, very weakly impressed distally and absent around upper margin of eyes; c) pronotum subrectangular or subtrapezoidal narrower at base, and lacking bevelled anterior angles (in Chaetocnema anterior angles are generally bevelled); d) ungual segment of hind tarsi generally swollen (Figs 9-10); e) vertex of head always distinctly and uniformly punctured (Figs 1-3) (in Chaetocnema vertex is often impunctate); f) labrum generally very short; g) elytral punctation arranged in regular rows; h) third hind tarsomere small, not or very slightly heartshaped (Fig. 9). Metafemoral spring (Fig. 18) attributable to Chaetocnema morpho-group (Furth \& Suzuki, 1998: 97); the extended arm of the dorsal lobe is short and depressed, ventral lobe with no recurved flange, dorsal edge straight and strongly angled down apically, and basal edge flat.

Taxonomy. The genus Pepila is part of a natural group of genera including: Chaetocnema Stephens, 1831, widespread in all the zoogeographical regions with over 300 species; Terpnochlorus Fairmaire, 1904, known from the Afrotropical (2 species) and Neotropical (1 species) regions; Carcharodis Weise, 1910, occurring in the Afrotropical region with at least four species; Collartaltica Bechyné, 1959, occurring in the Afrotropical region with at least three species; Biodontocnema Biondi, 2000, known from the South-West Africa with only one species; Seychellaltica Biondi, 2002, endemic to the Sey- 


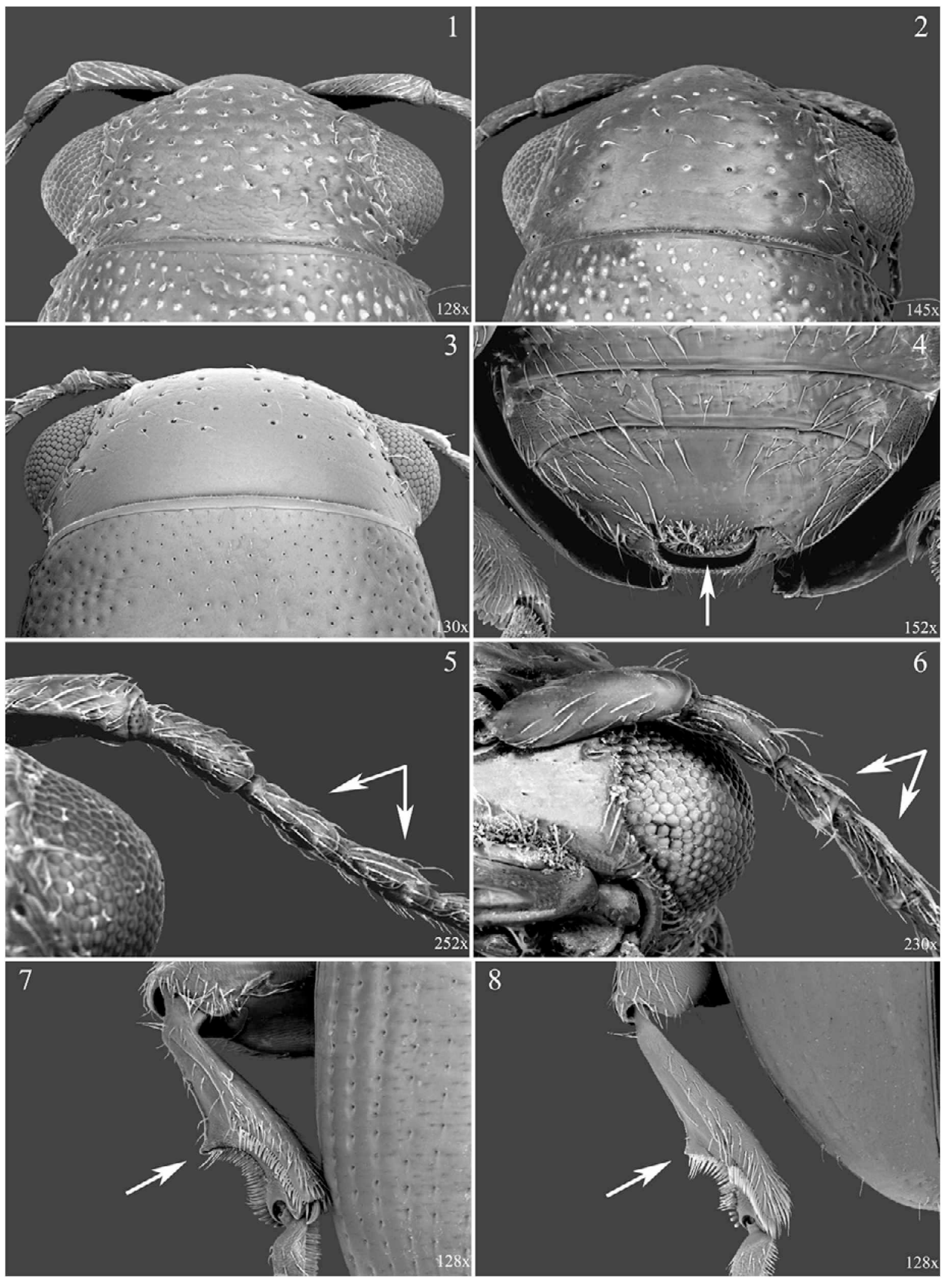

Figs 1-8: Morphological characters of the genus Pepila Weise. 1- head of P. megalopoides (Baly); 2 - ditto of P. uptoni n.sp.; 3 ditto of $P$. longifallica n.sp.; 4 - last sternite (white arrow) of male of $P$. longifallica n.sp.; 5 - antennal segments 3-4 (white arrows) of male of $P$. megalopoides (Baly); 6 - ditto of $P$. longifallica n.sp.; 7 - hind left tibia of $P$. megalopoides (Baly); $8-$ ditto of $P$. longifallica $\mathrm{n.sp}$.

chelles where there are at least four species (Biondi, 2002).
Phylogenetic relationships of Pepila and the above genera are discussed by Biondi (2002). 

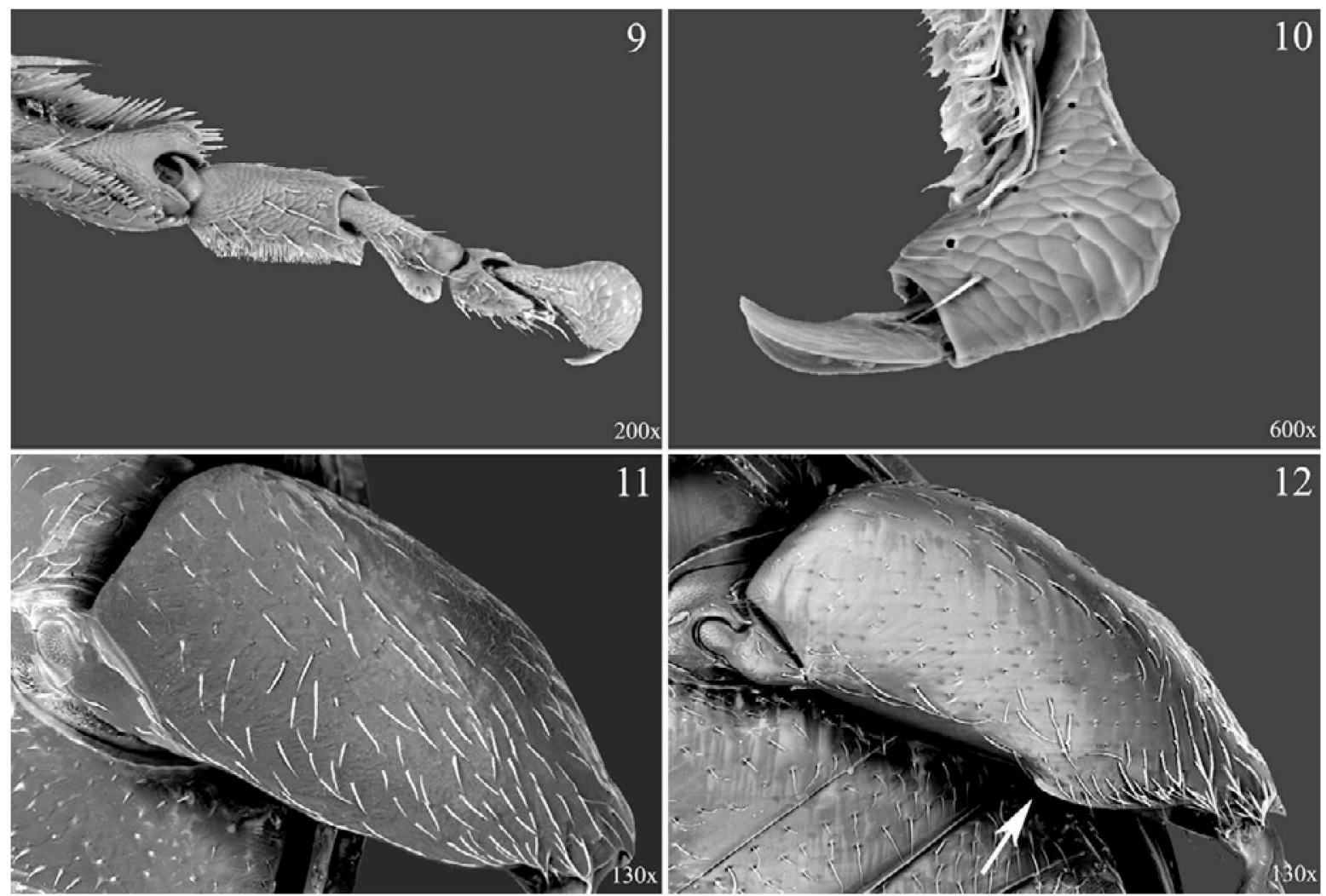

Figs 9-12: Morphological characters of the genus Pepila Weise. 9 - hind tarsomeres of $P$. megalopoides (Baly); 10 - hind apical tarsal segment of $P$. longifallica n.sp.; 11 - hind left femur in ventral view of $P$. megalopoides (Baly); 12 - ditto of $P$. longifallica n.sp.

In addition to $P$. megalopoides (Baly), the species listed below are transferred to the genus Pepila Weise. For each of them a lectotype is designated. The Fourth edition of the International Code of Zoological Nomenclature (ICZN, 1999) requires (Article 74.7.3) a lectotype designation to "contain an express statement of the taxonomic purpose of the designation". The purpose of the lectotype designations in this paper is to assure correct and consistent application of the names in the future. There is no reason to repeat this statement for each lectotype designation.

Pepila carinata (Baly, 1877) comb. n.

Chaetocnema carinata Baly, 1877: 174

Lectotype designation. Green card: "Chaetocnema / carinata / Baly / Western Australia" // white card: "Baly Coll." // red card (added by us): "Lectotype + / Chaetocnema carinata / Baly, 1877 / M. Biondi des. 2002" (BMNH).

\section{Pepila crassipennis (Blackburn, 1896) comb. n.}

Plectroscelis crassipennis Blackburn, 1896: 58, 66 Chaetocnema crassipennis: Bryant, 1923: 142 [erroneously synonymized with Dibolia ochracea Waterhouse, 1838: 135]

Lectotype designation. White card (1): “T-592" [South Australia, Eyre's Peninsula] // white card (2) (handwritten by G.E. Bryant): "Chaetocnema crassipen- nis, Blackb."// white card (3): "Blackburn coll. / 1910-236" // red card (added by us): "Lectotypus $q$ / Plectroscelis crassipennis / Blackburn, 1896 / M. Biondi des. 2002" (BMNH).

Pepila fuscomaculata (Baly, 1877) comb. n.

Chaetocnema fuscomaculata Baly, 1877: 174; Bryant, 1923: 141

Plectroscelis fuscomaculata: Blackburn, 1896: 58, 63

Lectotype designation. Green card: "Chaetocnema/ fuscomaculata / Baly / Western Australia" // white card: "Baly Coll." // red card (added by us) "Lectotypus o / Chaetocnema fuscomaculata / Baly, 1877 / M. Biondi des. 2002" (BMNH).

\section{Pepila hypocrita (Blackburn, 1896) comb. n.}

Plectroscelis hypocrita Blackburn, 1896: 58, 63

Lectotype designation. White card (1): "T-6094P" [Western Australia] // white card (2) (handwritten by G.E. Bryant): "Chaetocnema / hypocrita / Blackb."// white card (3): "Blackburn coll. / 1910-236" // red card (added by us): "Lectotypus +9 / Plectroscelis hypocrita / Blackburn, 1896 / M. Biondi des. 2002" (BMNH).

Pepila laticeps (Baly, 1877) comb. n.

Chaetocnema laticeps Baly, 1877: 315

Lectotype designation. Green card: "Chaetocnema/ laticeps / Baly / Western Australia" // white card: "Baly 


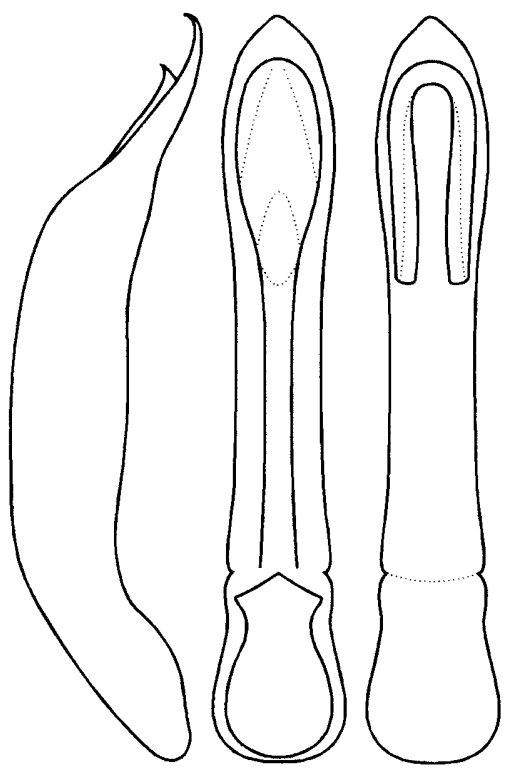

13

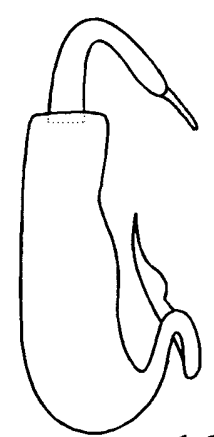

16
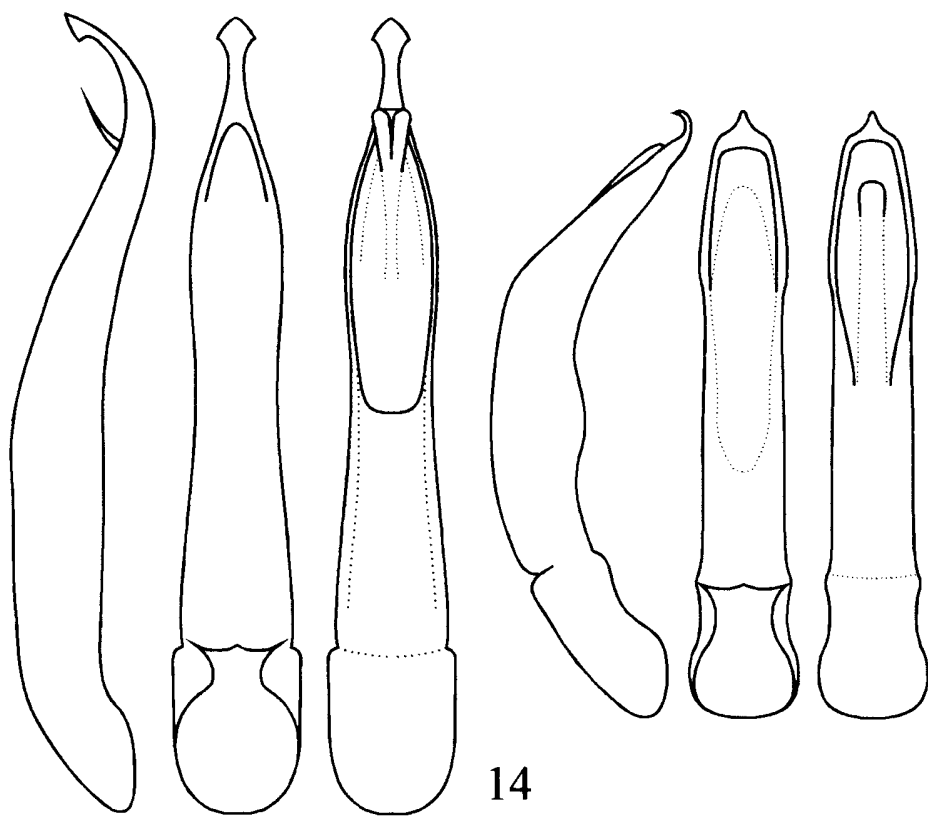

15

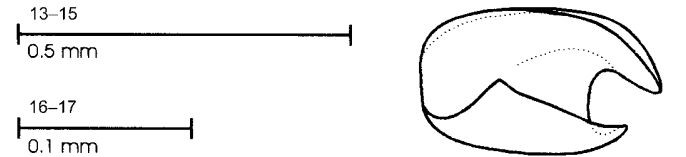

18

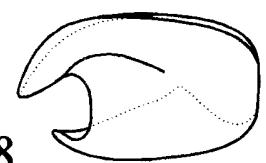

$\frac{18}{0.2 \mathrm{~mm}}$

Figs 13-18. Lateral, ventral and dorsal view of the median lobe of the aedeagus (13-15), spermatheca (16-17) and metafemoral spring (18) of Pepila species. 13 - P. megalopoides (Baly), Australia, Queensland, Archer Creek; 14 - P. longifallica n. sp., Australia, Northern Territory, McArthur River, holotype; 15 - P. uptoni n. sp., Australia, Northern Territory, McArthur River, holotype; 16 - P. megalopoides (Baly), Australia, Queensland, Rocky Creek; 17 - P. uptoni n. sp., Australia, Northern Territory, McArthur River, paratype; $18-P$. megalopoides (Baly).

Coll." // red card (added by us) "Lectotypus + / Chaetocnema laticeps / Baly, 1877 / M. Biondi des. 2002" $(\mathrm{BMNH})$.

\section{Pepila meyricki (Blackburn, 1896) comb. n.}

Plectroscelis meyricki Blackburn, 1896: 58, 66

Lectotype designation. White card (1): "T-1881" [Western Australia] // white card (2) (handwritten by G.E. Bryant): "Chaetocnema / meyricki, Blackb."// white card (3): "Blackburn coll. / 1910-236" // red card (added by us): "Lectotypus $q$ / Plectroscelis meyricki / Blackburn, 1896 / M. Biondi des. 2002" (BMNH).

\section{Pepila ochracea (Waterhouse, 1838) comb. n.}

Dibolia ochracea Waterhouse, 1838: 135

Lectotype designation. White card: "Dibolia ochracea / Waterhouse / Kg. George's Sound" [Australia, C. Darwin leg.] // red card (added by us): "Lectotypus $q$ / Dibolia ochracea / Waterhouse, 1838 / M. Biondi des. 2002" (BMNH).
Pepila pallidior (Blackburn, 1896) comb. $\mathbf{n}$. Plectroscelis pallidior Blackburn, 1896: 58, 65

Lectotype designation. White card (1): "T-8457" [South Australia] // white card (2) (handwritten by G.E. Bryant): "Chaetocnema / pallidior, Blackb."// white card (3): "Blackburn coll. / 1910-236" // red card (added by us): "Lectotypus + / Plectroscelis pallidior / Blackburn, 1896 / M. Biondi des. 2002” (BMNH).

\section{Pepila pygmaea (Waterhouse, 1838) comb. n.}

Dibolia pygmaea Waterhouse, 1838: 135

Lectotype designation. White card: "Dibolia pygmaea / Waterhouse / Kg. George's Sound" [Australia, C. Darwin leg.] // red card (added by us): "Lectotypus o / Dibolia pygmaea / Waterhouse, 1838 / M. Biondi des. 2002" (BMNH).

\section{Pepila quadraticollis (Blackburn, 1896) comb. n.}

Plectroscelis quadraticollis Blackburn, 1896: 58, 65 


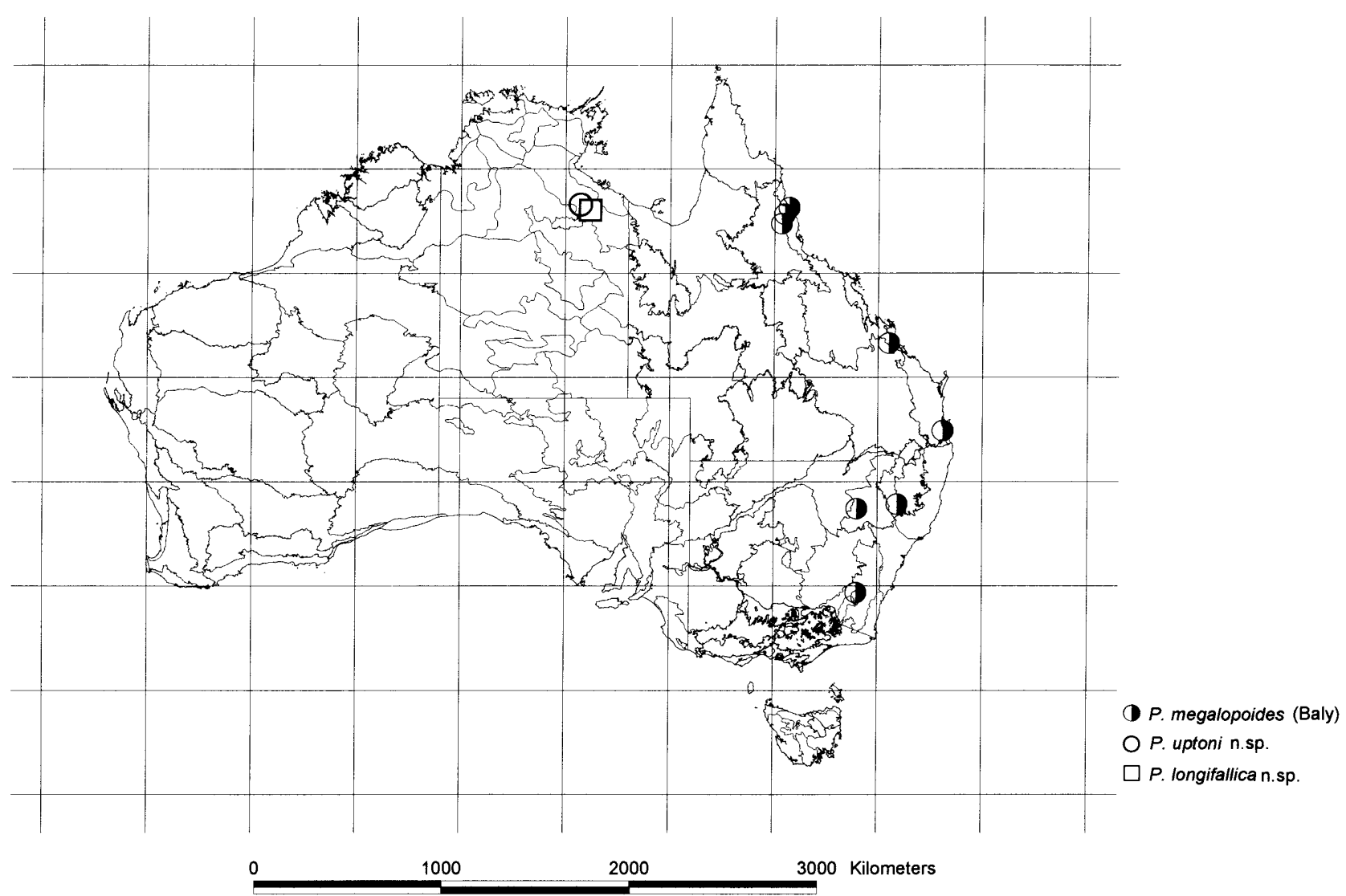

Fig. 19. Distribution of the Pepila megalopoides species-group in Australia.

Lectotype designation. White card (1): "T-508" [South Australia, Quorn] // white card (2) (handwritten by G.E. Bryant): "Chaetocnema / quadraticollis, Blackb."// white card (3): "Blackburn coll. / 1910-236" // red card (added by us): "Lectotypus $\% /$ Plectroscelis quadraticollis / Blackburn, 1896 / M. Biondi des. 2002" (BMNH).

\section{Pepila submetallescens (Baly, 1877) comb. n.}

Chaetocnema submetallescens Baly, 1877: 175

Plectroscelis longior Blackburn, 1896: 58-59 syn. n.

Lectotype designation. Chaetocnema submetallescens Baly: green card: "Chaetocnema / submetallescens / Baly / South Australia" [Gawler Town] // white card: "Baly Coll." // red card (added by us) "Lectotypus ô / Chaetocnema submetallescens / Baly, 1877 / M. Biondi des. 2002" (BMNH); Plectroscelis longior Blackburn: white card (1): "T-471" [South Australia, near Port Lincoln] // white card (2) (handwritten by G.E. Bryant): "Chaetocnema / longior, Blackb." // white card (3): "Blackburn coll. / 1910-236" // red card (added by us): "Lectotypus o / Plectroscelis longior / Blackburn, 1896 / M. Biondi des. 2002" (BMNH).

\section{Pepila tumbyensis (Blackburn, 1896) comb. n.}

Plectroscelis tumbyensis Blackburn, 1896: 58,63

Chaetocnema tumbyensis: Bryant, 1923: 141 [erroneously synonymized with Chaetocnema fuscomaculata Baly, 1877]
Lectotype designation. White card (1): "T-1185" [South Australia, Eyre's Peninsula, near Tumby] // white card (2) (handwritten by G.E. Bryant): "Chaetocnema / tumbyensis, Blackb."// white card (3): "Blackburn coll. / 1910-236" // red card (added by us): "Lectotypus o / Plectroscelis tumbyensis / Blackburn, 1896 / M. Biondi des. 2002" (BMNH).

Pepila waterhousei (Baly, 1877) comb. n.

Chaetocnema waterhousei Baly, 1877: 315

Plectroscelis waterhousei (Baly): Blackburn, 1896: 58

Lectotype designation. Green card: "Chaetocnema/ waterhousei / Baly / Western Australia" // white card: "Baly Coll." // red card (added by us) "Lectotypus $q /$ Chaetocnema waterhousei / Baly, 1877 / M. Biondi des. 2002" (BMNH).

Ecological remarks. From the ecological point of view, this Australian genus is practically unknown. However, its species seem to occur in different habitats, but mainly in temperate eucalyptus forests and other woodlands. Some species are associated with Myrtaceae (genera Angophora, Eucalyptus and Melaleuca) and others with Mimosaceae (genus Acacia) and Casuarinaceae (genus Casuarina) (Biondi \& D'Alessandro, in prep.).

\section{THE PEPILA MEGALOPOIDES SPECIES-GROUP}

This species-group includes $P$. megalopoides Weise, 1923, P. uptoni n.sp. and P. longifallica n.sp. Besides the 
same habitus, these three species share the following characters: 1) frontal sulci very short, clearly impressed only in the supra-antennal tract; 2) eyes roundish; 3 ) labrum with 8 preapical setae; 4) hind tibiae dorsally widely and deeply channelled (Figs 7-8); 5) hind tibiae with lateral emargination clearly and acutely prominent (Figs 7-8); 6) hind apical tarsal segment strongly swollen (Figs 9-10); 7) hind tibial socket wide.

\section{KEY TO SPECIES IN THE $P$. MEGALOPOIDES SPECIES-GROUP}

1. Head with vertex distally sparsely and weakly punctured (Fig. 3). Hind femora with evident emargination on ventral margin of apical third (Fig. 12). Fourth antennomere about $1 / 2$ longer than third (Fig. 6). Lateral emargination of hind tibiae less strongly prominent (Fig. 8). Median lobe of the aedeagus (Fig. 14) longer (LE/LAED $=1.49$ ), in ventral view with elongate and apically subrhomboidal distal part. Female unknown ............... P. longifallica n. sp.

- Head with vertex distally densely and strongly punctured (Figs 1-2). Hind femora with regularly arcuated ventral margin (Fig. 11). Lateral emargination of hind tibiae more strongly prominent (Fig. 7). Fourth antennal segment about as long as third (Fig. 5). Median lobe of aedeagus shorter $(\mathrm{LE} / \mathrm{LAED}>1.70)$, in ventral view distally lanceolate not subrhomboidal (Figs 13, 15) . . . . . . . . . . . 2

2. Size larger $(L B>2.40 \mathrm{~mm} ; \mathrm{LE}=>1.80 \mathrm{~mm})$. Head with vertex distally more densely and deeply punctured and with a clearly shagreened surface (Fig. 1). Dorsal integument generally darker, especially on head. Antennomeres 7-11 more robust and clearly blackened. Male with first anterior and middle tarsomere clearly dilated. Median lobe of aedeagus (Fig. 13) in ventral view rounded in distal part, apically with a very weakly prominent median small tooth; ventral sulcus narrow and basal half deeply impressed; in lateral view, median lobe less strongly curved

P. megalopoides (Baly)

- Size smaller (LB $<=2.40 \mathrm{~mm} ; \mathrm{LE}<1.80 \mathrm{~mm}$ ). Head with vertex distally more rarely and weakly punctured and with an almost smooth or slightly granulate surface (Fig. 2). Dorsal integument uniformly reddish brown. Antennomeres 7-11 more thin and weakly obscured. Male with first anterior and middle tarsomere weakly dilated. Median lobe of aedeagus (Fig. 15) in ventral view subrectangular in distal part, apically with a clearly prominent median small tooth; ventral sulcus wide and basal half weakly impressed; in lateral view, median lobe more strongly curved $\ldots . \ldots \ldots$. P. uptoni n. sp.

Pepila megalopoides (Baly, 1877)

Chaetocnema megalopoides Baly, 1877: 174

Pepila megalopoides: Weise, 1923: 126

Lectotype designation. Green card: "Chaetocnema / megalopoides / Baly / Rock Hampton" [Australia, Queensland] // white card: "Baly Coll." // red card (added by us) "Lectotypus $\delta$ / Chaetocnema megalopoides / Baly, 1877 / M. Biondi des. 2001" (BMNH).

Material examined. Australia: "Queensland, Rock Hampton" [23.22S 150.30E], “J. Baly Coll.", lectotype o (BMNH). Queensland: Redlynch [16.53S 145.41E], 12-20.viii.1938, Papuan-Australian Expedition B.M.1939-127, leg. R.G.Wind, 1 ex. (BMNH); Rocky Creek, $7 \mathrm{~m}$. N of Atherton [17.12S 145.28E], 3.v.1967, leg. D.H.Colless, 4 exx. (ANIC); Archer
Creek, $15 \mathrm{~km} \mathrm{~W}$ by S Ravenshoe [17.39S 145.20E], 20.v.1980, leg. I.D.Naumann \& J.C.Cardale, 4 exx. (ANIC); Silver Valley, 9/50 GB, Bequest 1976, leg. J.C.Brooks, 4 exx. (ANIC); Cedar Creek [27.57S 153.11E], mars, leg. E. Mjöberg, 1 ex. (NHRS). New South Wales: Tamworth [31.05S 150.55E], leg. A.M.Lea, 1 ex. (SAM); Warrumbungle NP NSW, Camp Pincham [31.18S 149.00E], 20-24.xi.1985, 10 bts., on Casuarina (A), leg. C.Reid, 3 exx. (ANIC); ibidem, on Casuarina (B), 10 exx. (ANIC); ibidem, 40 bts., on Angophora (B), 1ex. (ANIC); Cabbage Tree Creek, Canberra-Coast road, Casuarina Sands [35.19S 148.58E], 21.ii.1969, leg. E.B.Britton, W.Misko \& L.W.Simmons, 3 exx. (ANIC).

Redescription. $\delta \mathrm{LB}=2.61 \pm 0.07 \mathrm{~mm} ; \mathrm{LAN}=1.80 \pm$ $0.04 \mathrm{~mm} ; \mathrm{LP}=0.58 \pm 0.03 \mathrm{~mm} ; \mathrm{WP}=0.87 \pm 0.03 \mathrm{~mm}$; $\mathrm{LE}=1.91 \pm 0.04 \mathrm{~mm} ; \mathrm{WE}=1.17 \pm 0.03 \mathrm{~mm}$; LAED $=$ $0.99 \pm 0.03 \mathrm{~mm} ; \mathrm{LAN} / \mathrm{LB}=0.68 \pm 0.01 ; \mathrm{WP} / \mathrm{LP}=1.49 \pm$ $0.05 ; \mathrm{LE} / \mathrm{LP}=3.28 \pm 0.15 ; \mathrm{LE} / \mathrm{LAED}=1.91 \pm 0.03$. 9 : $\mathrm{LB}=2.80 \pm 0.12 \mathrm{~mm} ; \mathrm{LAN}=1.64 \pm 0.09 \mathrm{~mm} ; \mathrm{LP}=0.60$ $\pm 0.04 \mathrm{~mm} ; \mathrm{WP}=0.91 \pm 0.05 \mathrm{~mm} ; \mathrm{LE}=2.05 \pm 0.09 \mathrm{~mm}$; $\mathrm{WE}=1.27 \pm 0.10 \mathrm{~mm} ; \mathrm{LSP}=0.25 \pm 0.02 \mathrm{~mm} ; \mathrm{LAN} / \mathrm{LB}$ $=0.60 \pm 0.03 ; \mathrm{WP} / \mathrm{LP}=1.50 \pm 0.06 ; \mathrm{LE} / \mathrm{LP}=3.40 \pm$ $0.17 ; \mathrm{LE} / \mathrm{LSP}=8.19 \pm 0.56$.

Dorsal integument reddish brown, sometimes paler on the elytra, with evident metallic often gilt like reflection. Body elongate-elliptical, convex. Maximum pronotal width near middle; maximum elytral width in basal third in male and near middle in female.

Head with vertex proximally without punctures but finely shagreened; distal part of vertex and interocular space with strongly and uniformly impressed moderately dense punctation (Fig. 1); frontal tubercles absent; frontal sulci short but clearly impressed in the supra-antennal tract; interantennal space about as wide as length of antennomere 2; frontal carina moderately raised, apically rounded; eyes roundish, laterally weakly raised, of diameter about as long as length of antennomere 1; labrum very short, piceous, with eight preapical setae; maxillary palpi pale; antennae long about $3 / 4$ of the body length in male, shorter in female; antennomeres $1-5$ generally pale, 6-11 gradually and weakly browned; length of each antennal segment generally proportional to numerical sequences 99:47:52:49:45:46:53:58:67:59:93 ( $\delta)$ and 78:45:41:39:40:58:56:55:53:53:69 (q) $(1=0.003$ $\mathrm{mm})$.

Pronotum moderately transverse, subrectangular, laterally weakly rounded; lateral and basal margin clearly bordered; anterior angles with dentiform prominence apically subrounded; posterior angles obtuse, subrounded; punctation strongly and uniformly impressed on smooth surface.

Elytra elongate, entirely covering pygidium, laterally rounded; punctation arranged in 9 regular rows (+ 1 sutural interrupted generally at basal fourth), formed by large punctures strongly impressed on finely punctured surface; apical declivous more weakly punctured; lateral interstriae convex; humeral callus moderately prominent; macropterous. Scutellum small, subrectangular, with smooth surface.

Legs entirely reddish brown, often with hind femora and sometimes also anterior and middle femora more or less darkened; inner side of hind femora not emarginated 
(Fig. 11); hind tibiae widely and clearly channelled dorsally, with lateral emargination strongly and acutely prominent (Fig. 7); hind tibial socket wide; hind tibial apical spur short, robust, from reddish to brown, inserted on inner side of tibial apex; first hind tarsomere wider than third, subrectangular (Fig. 9); hind apical tarsal segment strongly swollen (Fig. 9); first anterior and middle tarsomere in male clearly more dilated than in female.

Ventral surface reddish brown generally with darker metasternum. Last sternite laterally with weak and sparse punctation, medially not punctured with smooth surface; apical margin in male with evident wide subrectangular or semicircular prominence, absent in female.

$\delta$ : median lobe of aedeagus elongate (Fig. 13), with very finely shagreened surface; in ventral view, tapered from base to apical fourth and distally lanceolate; apex acute with evident median subtruncate small tooth; ventral sulcus wide and apical half medially impressed, and basal half strongly narrowed; dorsal sulcus visible apically; dorsal ligula narrow, laterally parallel, apically raised; in lateral view, basal quarter of median lobe clearly bent, then more or less straight towards base; apex strongly curved in dorsal direction.

: spermatheca (Fig. 16) with basal part elongate, subcylindrical; apical part not globose or distinct from collum, with evident elongate appendix; ductus with two narrow half-coils.

Distribution. Australia: Queensland, New South Wales.

Host plants. This species was collected on plants of the genera Casuarina (fam. Casuarinaceae) and Angophora (fam. Myrtaceae).

\section{Pepila uptoni n. sp.}

Description. Holotype $(\delta)$ : dorsal integument reddish brown, slightly paler elytra, with evident metallic gilt like reflection. Body elongate-elliptical, convex ( $\mathrm{LB}=2.27$ $\mathrm{mm}$ ). Maximum pronotal width in middle: $0.72 \mathrm{~mm}$; maximum elytral width in basal third: $0.95 \mathrm{~mm}$.

Head with vertex proximally without punctures, almost smooth; distal part of vertex and interocular space with sparse, moderately impressed and uniformly distributed punctation (Fig. 2); frontal tubercles not distinct; frontal sulci short but deeply impressed in supra-antennal tract; interantennal space about as wide as length of antennomere 2; frontal carina weakly raised, apically rounded; eyes roundish, laterally strongly prominent, diameter about as long as length of antennomere 1; labrum very short, piceous, with eight preapical setae; maxillary palpi pale; antennae little longer than half body length (LAN = $1.37 \mathrm{~mm}$; $\mathrm{LAN} / \mathrm{LB}=0.60$ ); antennomeres $1-5$ pale and 6-11 grade to pale brown; length of each antennal segment proportional to numerical sequence $86: 44: 31: 41: 45: 47: 49: 49: 50: 50: 70(1=0.003 \mathrm{~mm})$.

Pronotum moderately transverse $(\mathrm{LP}=0.47 \mathrm{~mm}$; $\mathrm{WP} / \mathrm{LP}=1.53$ ), subrectangular, laterally weakly rounded; lateral and basal margin clearly bordered; anterior angles with dentiform prominence apically subrounded; posterior angles obtuse, subrounded; punctation strongly and uniformly impressed on smooth surface.
Elytra elongate $(\mathrm{LE}=1.50 \mathrm{~mm} ; \mathrm{LE} / \mathrm{LP}=3.16)$, entirely covering pygidium; laterally weakly rounded; punctation arranged in 9 regular rows (+ 1 sutural interrupted at basal fourth); large and strongly impressed punctulation on smooth surface; apical declivous less strongly punctured; lateral interstriae subconvex; humeral callus moderately prominent; macropterous. Scutellum small, halfroundish, with smooth surface.

Legs with tibiae and tarsi pale; anterior and middle femora more or less obscured; hind femora blackened with metallic gilt reflection; ventral margin of hind femora not emarginated; hind tibiae dorsally widely and distinctly channelled with lateral emargination strongly prominent; hind tibial socket wide; hind apical tibial spur short, robust, reddish, inserted on inner side of tibial apex; first hind tarsomere wider than third, subrectangular; hind ungual segment clearly swollen; first anterior and middle tarsomere dilated, subtriangular.

Ventral surface reddish brown with blackened metasternum. Last sternite laterally very weakly and less densely punctured; medially with almost smooth surface, not punctured; apically with an evident median and wide semicircular prominence.

Median lobe of aedeagus elongate $(\mathrm{LAED}=0.82 \mathrm{~mm}$; LE/LAED = 1.82) (Fig. 15), with almost smooth surface; in ventral view clearly lanceolate with apical third narrowed; apex rounded with median small tooth clearly prominent; ventral sulcus wide and distinctly impressed on apical $2 / 3$; dorsal sulcus present on apical half; dorsal ligula apically widely rounded and raised; apical third of median lobe bent in lateral view and with an evident hump on inner side; apex clearly curved in dorsal direction.

Paratypes. $\delta$ : $\mathrm{LB}=2.32 \mathrm{~mm} ; \mathrm{LAN}=1.50 \mathrm{~mm} ; \mathrm{LP}=$ $0.47 \mathrm{~mm} ; \mathrm{WP}=0.75 \mathrm{~mm} ; \mathrm{LE}=1.55 \mathrm{~mm} ; \mathrm{WE}=1.00$ $\mathrm{mm} ; \mathrm{LAED}=0.82 \mathrm{~mm} ; \mathrm{LAN} / \mathrm{LB}=0.64 ; \mathrm{WP} / \mathrm{LP}=1.58$; $\mathrm{LE} / \mathrm{LP}=3.26 ; \mathrm{LE} / \mathrm{LAED}=1.88$. $q: \mathrm{LB}=2.32 \pm 0.08$ $\mathrm{mm} ; \mathrm{LAN}=1.37 \pm 0.01 \mathrm{~mm} ; \mathrm{LP}=0.49 \pm 0.02 \mathrm{~mm} ; \mathrm{WP}$ $=0.74 \pm 0.02 \mathrm{~mm} ; \mathrm{LE}=1.61 \pm 0.02 \mathrm{~mm} ; \mathrm{WE}=1.01 \pm$ $0.05 \mathrm{~mm} ; \mathrm{LSP}=0.22 \pm 0.01 \mathrm{~mm} ; \mathrm{LAN} / \mathrm{LB}=0.60 \pm 0.04$; $\mathrm{WP} / \mathrm{LP}=1.51 \pm 0.02 ; \mathrm{LE} / \mathrm{LP}=3.31 \pm 0.08 ; \mathrm{LE} / \mathrm{LSP}=$ $7.16 \pm 0.08$. Female generally larger, with $1^{\text {st }}$ tarsomere of anterior and middle legs narrower. Length of each antennal segment generally proportional to numerical sequence $80: 43: 32: 36: 45: 51: 47: 48: 49: 48: 69(1=0.003$ $\mathrm{mm}$ ).

Spermatheca with basal part elongate (Fig. 17), subcylindrical; apical part not globose, not distinct from collum and with evident elongate appendix; ductus with two narrow half-coils.

Diagnosis. This new species is very close to P. megalopoides but is easily distinguished by: smaller size (LB < $2.40 \mathrm{~mm}$ ); more rarely and weakly impressed punctation on head (Fig. 2); different shape of the median lobe of the aedeagus (Fig. 15).

Type material. Holotype $\delta$, Australia: "Northern Territory, McArthur River, $14 \mathrm{~km} \mathrm{~S}$ by W of Cape Crawford, 16.47S 135.45E, 25.x.1975, leg. M.S.Upton" (ANIC). Paratypes: Aus- 
tralia: same locality, date and collector as the holotype, 1 and 2 \& (ANIC).

Etymology. This species is named after Murray Scott Upton (Australia, Brisbane), its collector.

Distribution. Australia: Northern Territory.

Host plants. There is no information on its host plants. Pepila longifallica n. sp.

Description. Holotype $(\approx)$ : dorsal integument reddish brown paler on elytra, with evident gilt metallic reflection; body elongate-elliptical, convex ( $\mathrm{LB}=2.37$ $\mathrm{mm}$ ). Maximum pronotal width near middle: $0.82 \mathrm{~mm}$; maximum elytral width at basal third: $1.07 \mathrm{~mm}$.

Head with vertex proximally without punctures, almost smooth; distal part of vertex and interocular space with very little densely and weakly impressed punctation (Fig. 3); frontal tubercles absent; frontal sulci short, clearly impressed in supra-antennal tract; interantennal space about as wide as length of antennomere 5; frontal carina moderately raised, apically rounded; eyes roundish, laterally prominent, diameter about as long as length of antennomere 1; labrum very short, piceous, with eight preapical setae; maxillary palpi pale; antennae long, little more than half body length (LAN $=1.57 \mathrm{~mm}$; LAN $/ \mathrm{LB}=$ 0.66 ); antennomeres $1-6$ pale, $7-11$ grade to very pale brown; length of each antennal segment proportional to numerical sequence $89: 39: 30: 52: 45: 47: 49: 73: 70: 63: 83$ (1 $=0.003 \mathrm{~mm}$ ).

Pronotum moderately transverse $(\mathrm{LP}=0.57 \mathrm{~mm}$; WP/LP $=1.13$ ), subrectangular but basally slightly narrower; laterally weakly rounded; lateral and basal margin clearly bordered; anterior angles with dentiform prominence apically subrounded; posterior angles obtuse; punctation clearly impressed, formed by punctures of different size uniformly distributed on smooth surface.

Elytra elongate $(\mathrm{LE}=1.67 \mathrm{~mm} ; \mathrm{LE} / \mathrm{LP}=2.91)$, entirely covering pygidium; laterally weakly rounded; punctation arranged in 9 regular rows ( +1 sutural interrupted at basal fourth), strongly impressed on smooth surface; apical declivous more weakly punctured; lateral interstriae almost flat; humeral callus moderately prominent; macropterous. Scutellum small, subtriangular, with smooth surface.

Legs entirely pale but with darkened hind femora partially with gilt metallic reflection; ventral margin of hind femora with evident emargination on apical third (Fig. 12); hind tibiae dorsally widely and deeply channelled, with clearly and acutely prominent lateral emargination (Fig. 8); hind tibial socket wide; hind apical spur short, robust, reddish, inserted on inner side of tibial apex; first hind tarsomere wider than third, subrectangular; hind apical tarsal segment strongly swollen (Fig. 10); first anterior and middle tarsomere clearly dilated.

Ventral surface reddish brown with darkened metasternum. Last sternite laterally with weak and sparse punctation; medially not punctured, with smooth surface; apical margin with evident wide subrectangular prominence in middle (Fig. 4).

Median lobe of aedeagus elongate $(\mathrm{LAED}=1.12 \mathrm{~mm}$; $\mathrm{LE} / \mathrm{LAED}=1.49$ ) (Fig. 14), with almost smooth surface; in ventral view, laterally narrowed in middle; distal part elongate, apically subrhomboidal; ventral sulcus not visible; apical half of dorsal sulcus widely and deeply impressed; dorsal ligula narrow, distal margin apically clearly raised and medially weakly incised; in lateral view, median lobe apically strongly curved in dorsal direction and basally almost straight.

Diagnosis. $P$. longifallica $\mathrm{n}$.sp. is the most distinct species within this group. The male (the female is unknown) is easily distinguished on the basis of the following characteristics: head with very rare and weakly impressed punctation (Fig. 3); apical third of hind femora with evident emargination on ventral margin (Fig. 12); lateral emargination of hind tibiae less strongly prominent (Fig. 8); fourth antennal segment about 11/2 length of third (Fig. 6); distal part of median lobe of aedeagus in ventral view elongate and apically subrhomboidal (Fig. 14).

Type material. Holotype $\delta$, Australia: "Northern Territory: McArthur River, $14 \mathrm{~km} \mathrm{~S}$ by W of Cape Crawford, 16.47S 135.45E, 25.x.1975, leg. M.S.Upton" (ANIC).

Etymology. The name of this species refers to the median lobe of the aedeagus, which is comparatively longer than in other species of this group.

Distribution. Australia: Northern Territory.

Host plants. There is no information on its host plants.

ACKNOWLEDGEMENTS. We are very grateful to colleagues who enabled us to study the valuable material preserved in their respective institutions: E. Matthews (SAM), S.L. Shute (BMNH), B. Viklund (NHRS) and T.A. Weir (ANIC).

\section{REFERENCES}

BALY J.S. 1877: Descriptions of a new genera and of uncharacterized species of Halticinae. Trans. Entomol. Soc. Lond. 1877: 157-184, 283-323.

BeCHYNÉ J. 1959: Observations sur les Alticides Africains des collections de 1'Institut Royal des Sciences Naturelles de Belgique (Coleoptera, Phytophaga). Bull. Mens. Soc. Linn. Lyon 32: $235-239$.

BIONDI M. 2000 : Biodontocnema brunnea n.gen. and n.sp. from South Africa (Coleoptera: Chrysomelidae: Alticinae). Coleopt. Bul. 54: 347-350.

BIONDI M. 2002: Chaetocnema Stephens and related genera: a systematic revision, with description of a new genus from the Indian Ocean (Seychelles) (Coleoptera: Chrysomelidae). Ital. J. Zool. 69: 355-366.

BLACKBURN T. 1896: Further notes on Australian Coleoptera, with descriptions of new genera and species. Trans. R. Soc. S. Aust. 20: 35-109.

BryanT G.E. 1923: Notes on synonymy in the Phytophaga (Coleoptera). Ann. Mag. Nat. Hist. 12: 130-147.

FAIRMArRE L. 1904: Materiaux pour la faune coléopterique malgache. 18e note. Annls Soc. Entomol. Belg. 48: 225-276.

Furtu D.G. \& Suzuki K. 1998: Studies of Oriental and Australian Alticinae genera based on the comparative morphology of the metafemoral spring, genitalia, and hind wing venation. In: Biondi M., Daccordi M. \& Furth D.G. (eds): Proceedings of the Fourth International Symposium on the Chrysomelidae. Proceedings of XX I.C.E. Firenze, 1996, Museo Regionale di Scienze Naturali, Torino, pp. 91-124.

ICZN (International Commission on Zoological Nomenclature). 1999: International Code of Zoological Nomenclature. Fourth 
Edition. International Trust for Zoological Nomenclature, London, xxix $+306 \mathrm{pp}$.

STEPHENS J. 1831: Illustrations of British Entomology. IV. Mandibulata. Baldwin \& Cradock, London, $408 \mathrm{pp}$.

Waterhouse C.O. 1838: Descriptions of some of the Insects brought to this country by C. Darwin Esq. Trans. Entomol. Soc. Lond. 2: 131-135.
WeISE J. 1910: Chrysomelidae von Madagaskar, den Comoren, und den Inseln (Ostafrikas). In: S. Voeltzkow (ed.): Reise in Ostafrika in den Jahren 1903-1905, Band II, Heft 5. E. Schweizerbartsche Verlagsbuchhandlung, Nägele \& Dr. Sproesser, Stuttgart, pp. 419-506.

WeISE J. 1923: Chrysomeliden und Coccinelliden aus Queensland. Ark. Zool. 15: 1-150.

Received June 17, 2002; revised September 2, 2002; accepted October 29, 2002 